All letters are subject to editing and may be shortened. Letters should be sent to the BJGP office by e-mail in the first instance, addressed to

journal@rcgp.org.uk (please include your postal address). Alternatively, they may be sent by post as an MS Word or plain text version on CD or DVD. We regret that we cannot notify authors regarding publication. Letters not published in the Journal may be posted online on our Discussion Forum. For instructions please visit: http://www.rcgp.org.uk/bjgp-discuss

\section{Travelling in Palestine}

I was interested in Dr Shaw's response' to my essay, describing our family's personal experience of passing through an Israeli checkpoint. ${ }^{2}$ We did not simply have to 'wave a British passport'.

I also made it clear that we have friends in both Palestine and Israel who are committed to a peaceful coexistence. As in all situations, the more individual contact there is between people of different backgrounds and cultures, and the more we question unhelpful stereotypes based on fear, the more hope there is for peace.

\section{Lesley Morrison,}

14 Kingsmeadows Road,

Peebles EH45 9EN. E-mail:

Lesley.Morrison.teviot@borders.scot.nhs.uk

\section{REFERENCES}

1. Shaw F. Palestine. Br J Gen Pract 2009; 59(561): 290-291.

2. Morrison L. Stories from Palestine and Israel. $\mathrm{Br} \mathrm{J}$ Gen Pract 2009; 59(560): 216-217.

DOI: 10.3399/bjgp09X420662

\section{Improving the sensitivity of the Dutch guidelines for case finding in osteoporosis}

In a previous paper ${ }^{1}$ we reported on the poor validity of the Dutch case finding method for GPs to identify patients with osteoporosis for Dual energy X-ray absorptiometry (DXA) measurement (NHG guidelines for osteoporosis ${ }^{2}$ ), as sensitivity was $19.5 \%$ and positive predictive value (PPV) was $18.6 \% .^{2}$ We suggested that a more appropriate tool is needed to apply case finding for osteoporosis.

The problem, however, is that many GPs have poor knowledge of the different case finding methods that are available. ${ }^{3}$ Instead of designing a new method, one might rather evaluate alternative usage of current guidelines to achieve better results. Hence the aim of this research letter is to investigate alternative usage of the Dutch guidelines to select osteoporosis patients for DXA.

As we discussed in our previous paper, the poor validity of the Dutch guidelines might result from the definition of the weighted scores. Therefore, we performed receiver operating characteristic (ROC) curves to evaluate whether ' 4 ' is the best cut-off score to be used. As only 64 males were included, data analyses were performed of 345 females. Osteoporosis was diagnosed according to the World Health Organization $(\mathrm{WHO})$ guidelines $(\mathrm{T}$ score $\leq$
$-2.5 \mathrm{SD}$ ) and, in addition, according to the Dutch guidelines, using the WHO criteria in patients younger than 70 years and Z-scores in patients over 70 years $(\leq-1.0 \mathrm{SD})$ to define osteoporosis. Osteopenia was not defined within this age group. We calculated sensitivity, specificity, and predictive value of the guidelines using varying cut-offs; $95 \%$ confidence intervals were calculated using binomial expansion. Statistical analyses were performed using SPSS (version 16.0) software. The ROC curve, as well as the results of sensitivity, specificity, and predictive value (Table 1), showed that the best cut-off for the current Dutch instrument is ' 1 '. Using this cut-off and the WHO criteria of osteoporosis, sensitivity improved to $88 \%$, specificity was $40 \%$, PPV $14 \%$, and NPV $97 \%$. Slightly lower values were calculated if DXA outcome was based on the Dutch criteria of osteoporosis (using $Z$-scores in patients aged $\geq 70$ years.

If the optimal threshold is ' 1 ', it can be concluded that the weighted scores are of no added value. Moreover, it implies that screening is always recommended above the age of 60 years. In contrast, the US guidelines have recommended

Table 1. Sensitivity, specificity, positive predictive value (PPV), and negative predictive value (NPV) of the original Dutch osteoporosis guidelines in 345 women.

\begin{tabular}{lccccc} 
Cut-off & Criteria $^{a}$ & $\begin{array}{c}\text { Sensitivity, \% } \\
(95 \% \mathrm{Cl})\end{array}$ & $\begin{array}{c}\text { Specificity, \% } \\
(95 \% \mathrm{Cl})\end{array}$ & $\begin{array}{c}\mathrm{PPV}^{\mathrm{b}}, \% \\
(95 \% \mathrm{Cl})\end{array}$ & $\begin{array}{c}\text { NPV }, \% \\
(95 \% \mathrm{Cl})\end{array}$ \\
\hline 1 & WHO & $88(77-99)$ & $40(35-46)$ & $14(9-18)$ & $97(95-99)$ \\
& Dutch & $83(68-98)$ & $39(34-45)$ & $9(5-13)$ & $97(95-99)$ \\
\hline 2 & WHO & $63(47-80)$ & $67(62-73)$ & $17(10-24)$ & $95(91-99)$ \\
& Dutch & $50(30-70)$ & $65(60-71)$ & $10(5-15)$ & $95(91-99)$ \\
\hline 4 & WHO & $36(20-35)$ & $88(84-91)$ & $24(12-35)$ & $93(86-100)$ \\
& Dutch & $29(11-47)$ & $83(79-88)$ & $14(4-23)$ & $93(86-100)$ \\
\hline & WHO & $21(7-35)$ & $90(87-93)$ & $18(6-31)$ & $92(83-100)$ \\
& Dutch & $20(10-37)$ & $90(86-93)$ & $13(3-24)$ & $94(86-102)$ \\
\hline
\end{tabular}

aWHO-criteria: DXA outcome is always based on T-scores. Dutch-criteria: DXA outcome is based on Tscores if age $<70$ years and on $Z$-scores of age $\geq 70$ years. 